\title{
El comercio exterior y el crecimiento económico de México
}

Foreign Trade and

Economic Growth in Mexico

Recibido: 4 de septiembre de 2013

Evaluado: 3 de octubre de 2013

Aceptado: 10 de octubre de 2013

\author{
José Antonio Domínguez González (México) \\ Maestro en Administración. Universidad de Guadalajara \\ Correo electrónico: dantonio@cucea.udg.mx
}

\section{Resumen}

El objetivo del presente artículo es tratar de explicar por qué en algunos países, como México, el aumento en las exportaciones como resultado de una depreciación en el tipo de cambio viene a disminuir a largo plazo el crecimiento económico del país contradiciendo así la teoría clásica keynesiana, que dice que la depreciación del tipo de cambio provocará un incremento en las exportaciones, así como un decremento en las importaciones, mejorando de esa manera la balanza comercial del país, y provocando un aumento en el producto interno bruto. El método de investigación que se utiliza es de tipo deductivo, pues toma como base la teoría económica clásica, para tratar de entender el caso específico de México.

Palabras clave: depreciación, crecimiento, importaciones, exportaciones, modelo econométrico.

\begin{abstract}
This article aims to explain why in some countries, as Mexico, the increase of exports -as the result of depreciation in the type of exchange rate- decreases in the long term the economic growth of the country, thus contradicting classic Keynesian theory. This theory establishes that depreciation of the type of exchange rate leads to an increase in exports and to a decrease in imports, improving the commercial balance of the country and increasing the gross internal product. The research method used is deductive because it is based on the classic economic theory in order to try to understand the specific case of Mexico.
\end{abstract}

Keywords: depreciation, growth, imports, exports, econometric model. 


\section{Introducción}

La teoría económica tradicional basada en la clásica teoría keynesiana nos enseña que un aumento en el tipo de cambio de un país, debido a una depreciación de la moneda local, provocará un incremento en las exportaciones, así como una disminución en las importaciones, mejorando así la balanza comercial del país, trayendo como resultado final un aumento en el producto interno bruto (PIB) del país.

La demanda agregada de un país puede descomponerse en cuatro factores que representan diferentes tipos de demanda, como son el consumo de las familias en bienes y servicios, la inversión interna en nuevos activos reales, como maquinaria, edificios, casa e inventarios, el gasto del Gobierno en bienes y servicios y las exportaciones netas de bienes y servicios, o sea, la diferencia entre las exportaciones y las importaciones del país.

De acuerdo con la teoría económica tradicional, un incremento en el tipo de cambio de un país, debido a una depreciación de la moneda local, estimularía a los habitantes de dicho de país a incrementar la exportación de bienes y servicios, porque de esa manera saldrían beneficiados económicamente. Del mismo modo, la depreciación de la moneda local del país haría que los habitantes disminuyeran el consumo de bienes y servicios importados porque estos verían incrementado de inmediato su costo de adquisición. El resultado final sería un incremento en su balanza comercial, puesto que esta matemáticamente es igual a la diferencia entre las exportaciones y exportaciones de bienes y servicios de dicho país. Finalmente, de acuerdo con esta teoría económica, el incremento de la balanza comercial traería aparejado un incremento multiplicado en el PIB. Esto debido a un efecto multiplicador, cuyo valor es obtenido al dividir 1/1-PMI.

PMI significa propensión marginal por importar, y se obtiene al dividir el cambio en la cantidad importada entre el cambio en el PIB.

Sin embargo, el análisis empírico de los datos económicos de México en un determinado periodo nos muestra que la mencionada teoría keynesiana no siempre se da en la práctica. Al elaborar modelos econométricos con los datos económicos mencionados, nos encontramos resultados que contradicen lo expuesto.

\section{Marco teórico}

Según Pugel (2004), siguiendo la economía keynesiana tradicional, la demanda agregada puede descomponerse en cuatro aspectos que representan diferentes tipos de demanda: el consumo de las familias en bienes y servicios (C); la inversión interior en nuevos activos reales, como maquinaria, edificios, casa e inventarios (I); el gasto de la administración en bienes y servicios $(\mathrm{G})$, y las exportaciones netas de bienes y servicios o sea $(X-M)$. El equilibrio ocurrirá cuando la producción nacional $(\mathrm{Y}$, el PNB) sea igual a la demanda deseada de bienes y servicios producidos internamente: $\mathrm{Y}=\mathrm{DA}=\mathrm{C}+\mathrm{I}+\mathrm{G}+(\mathrm{X}-\mathrm{M})$.

De acuerdo con esto, el aumento en la balanza comercial, como son las exportaciones netas, debe provocar un aumento en el PIB. En México - como se demostrará más adelante- esta teoría no siempre se ha dado en la vida real. 
De acuerdo con Cortés Fregoso (2009), la explicación de este fenómeno se encuentra en la poca difundida ley de Thirlwall, ley creada por Anthony P. Thirlwall.

Thirlwall postula que a largo plazo la tasa de crecimiento observada (y) es igual a la tasa de crecimiento consistente con el equilibrio de la BP $\left(\mathrm{y}_{\mathrm{B}}\right)$ :

$$
\mathrm{yB}=\mathrm{y}=(€ / \pi) \mathrm{z}=(1 / \pi) \mathrm{x}
$$

Esta es la ley fundamental del crecimiento de A. P. Thirlwall que establece que a largo plazo la expansión de una economía... se haya restringida por el equilibrio de la cuenta corriente de la balanza de pagos (BPC) (Thirlwall, 2009, p. 13).

Es decir $\mathrm{y}=\mathrm{x} / \pi$

\section{Donde:}

$\mathrm{x}=$ Tasa de crecimiento del volumen de exportaciones

$€=$ Elasticidad ingreso de la demanda de exportaciones

$\pi=$ Elasticidad ingreso de la demanda de importaciones

$y=$ Tasa de crecimiento del ingreso nacional

$\mathrm{z}=$ Tasa de crecimiento del ingreso internacional

Algunos autores como García (2009), Loria (2001) y Guerrero (2003), se han interesado en analizar la ley de Thirlwall y estudiar su efecto práctico en México.

Loria (2001), en estudios empíricos, nos dice que para México se cumple esta ley en el sentido de que, debido a su alta dependencia de insumos importados, el desequilibrio comercial externo es el principal elemento que restringe el crecimiento y limita la utilización de los recursos físicos y humanos disponibles.
En México, se da la ley de Thirlwall, es decir, el incremento en las exportaciones incrementa a su vez el incremento de importaciones de insumos necesarios para llevar a cabo la producción de bienes. Como el incremento de las importaciones termina por superar al de las exportaciones, la balanza de pagos termina siendo deficitaria.

García (2009) dice que el crecimiento del producto puede ser aproximado por la tasa de crecimiento de las exportaciones dividido entre la elasticidad ingreso de las importaciones.

Guerrero (2003) asegura, en un análisis empírico de México, que como el incremento de la elasticidad ingreso de las exportaciones fue menor que el de la elasticidad ingreso de las importaciones la proporción cayó en el periodo 1987-2000 respecto del periodo precedente de 19401981. Esto representa un grave despropósito de la política económica instrumentada en el mercado del nuevo modelo implantado en nuestro país.

Esta situación explica por qué la crisis estructural de la producción alimentaria y las necesidades de bienes intermedios y de capital para apoyar el crecimiento generan un incremento más rápido del subsector importador que del subsector exportador, y el sistema económico vuelve a reproducir el déficit recurrente aunque en menor proporción que en el pasado.

Existe en México una elasticidad positiva de las exportaciones, es decir, las depreciaciones de la moneda han ayudado a mejorar la balanza comercial, pero no se ha cumplido la teoría del multiplicador, o sea, el incremento en el PIB no ha sido acorde con los cambios en el sector exportador. 


\section{Método}

El método utilizado en el presente trabajo es el método deductivo, el cual parte de una teoría para llegar a analizar un fenómeno particular. Después de analizar las teorías conocidas relacionadas con el fenómeno en cuestión, se pasará a un método empírico para tratar de encontrar relaciones entre las variables y establecer patrones de conducta. Finalmente, se elaborarán modelos econométricos para llevar a cabo los análisis necesarios que nos ayuden a encontrar las causas del fenómeno estudiado, así como sugerir propuestas para su solución.

Utilizando datos empíricos de México de 1996 a 2010, se procederá a la elaboración de un modelo econométrico basado en la teoría keynesiana.

Este modelo econométrico tiene la siguiente representación matemática:

$$
\begin{gathered}
\mathrm{Y}=\alpha+\beta 1+\beta 2+€ \partial \mathrm{Y} / \partial \beta 1>0, \mathrm{y} \\
\partial \mathrm{Y} / \partial \beta 2>0
\end{gathered}
$$

Donde:

$$
\begin{gathered}
Y=\text { Producto interno bruto } \\
\beta 1=\text { Balanza comercial } \\
\beta 2=\text { Tipo de cambio }
\end{gathered}
$$

Este modelo propone que el valor del PIB de México depende de manera positiva de la balanza comercial y del tipo de cambio. Es decir que un incremento en la balanza comercial o en el tipo de cambio de México provocará un incremento en el PIB del país.
Esto debido a que de acuerdo con la teoría clásica keynesiana, el incremento del tipo de cambio provocará que los habitantes se sientan estimulados a incrementar las exportaciones de bienes y servicios, así como a disminuir las importaciones por incrementarse su precio. El resultado final de este proceso sería un incremento en la balanza comercial, por ser esta la diferencia entre exportaciones e importaciones, así como el subsecuente incremento en el PIB.

Utilizando datos de la tabla 1, se obtuvieron los parámetros siguientes mediante el programa Eviews.

$$
\begin{gathered}
\mathrm{Y}=4319.921-0.059945 \mathrm{BC}+ \\
314.6206 \mathrm{TC}
\end{gathered}
$$

Donde:

$$
\begin{gathered}
\mathrm{Y}=\text { Producto interno bruto } \\
\mathrm{BC}=\text { Balanza comercial } \\
\mathrm{TC}=\text { Tipo de cambio }
\end{gathered}
$$

Independientemente de los valores reales de los parámetros, lo que llama la atención en esta ecuación son los signos que acompañan a tales parámetros. La anterior ecuación no concuerda con la tradicional teoría económica, el valor positivo de la variable $\mathrm{TC}$ es normal porque un aumento en TC incrementa las exportaciones, disminuye las importaciones y provoca que aumente la balanza comercial.

Lo que resulta irregular es que la variable $\mathrm{BC}$ tenga pendiente negativa. Porque esto significaría que un incremento en la balanza comercial haría disminuir el PIB, lo cual contradeciría lo señalado por la teoría económica tradicional. 
Tabla 1.

\begin{tabular}{|l|r|r|r|}
\hline Año & $\begin{array}{r}\text { PIB } \\
\text { (miles de } \\
\text { millones } \\
\text { de pesos) }\end{array}$ & $\begin{array}{c}\text { BC } \\
\text { (millones } \\
\text { de } \\
\text { dólares) }\end{array}$ & \multicolumn{1}{c|}{ TC } \\
\hline 1996 & 6427.559 & 6531 & 7.83 \\
\hline 1997 & 6898.433 & 624 & 8.08 \\
\hline 1998 & 7072.2 & -7742 & 10.02 \\
\hline 1999 & 7410.151 & -5583 & 9.46 \\
\hline 2000 & 7711.444 & -8003 & 9.49 \\
\hline 2001 & 7576.348 & -9954 & 9.67 \\
\hline 2002 & 7670.056 & -7916 & 10 \\
\hline 2003 & 7781.183 & -5623 & 10.78 \\
\hline 2004 & 8133.313 & -8811 & 11.28 \\
\hline 2005 & 8423.951 & -7586 & 10.77 \\
\hline 2006 & 8765.547 & -6133 & 10.89 \\
\hline 2007 & 9078.916 & -10074 & 10.92 \\
\hline 2008 & 8985.252 & -16700 & 10.6 \\
\hline 2009 & 8802.47 & -4681 & 15.2 \\
\hline 2010 & 9191.549 & -3009 & 12.2 \\
\hline & & & \\
\hline
\end{tabular}

Fuente: Modificado de Inegi y Banxico.

Tabla 2.

\begin{tabular}{|c|c|c|c|c|c|}
\hline Observaciones & 15 & \multicolumn{2}{|c|}{$\begin{array}{l}\text { Método: } \\
\text { mínimos } \\
\text { cuadrados }\end{array}$} & $\begin{array}{c}\text { Variable } \\
\text { dependiente } \\
\mathbf{Y}\end{array}$ & $\begin{array}{c}\text { Variables } \\
\text { independientes } \\
\text { ВС y TC }\end{array}$ \\
\hline Variable & Coeficiente & Error estár & dar & Estadístico t & Probabilidad \\
\hline Origen & -.050945 & & .028411 & -2.109894 & .0565 \\
\hline$B C$ & 314.6206 & & 84.93970 & 3.704046 & .0030 \\
\hline $\mathrm{TC}$ & 4319.921 & & 865.9009 & 4.988932 & .0003 \\
\hline$R^{2}$ & & 0.674471 & Media var & depend & 7995.22 \\
\hline $\mathrm{R}^{2}$ ajustada & & 0.620216 & Estadístico & & 12.43153 \\
\hline Durbin Watson & & 1.459315 & Probabilid & $\operatorname{ad~F}$ & 0.001190 \\
\hline
\end{tabular}

Nota: Resultado obtenido con el programa Eviews.

Fuente: Elaboración propia. 


\section{Resultados}

De acuerdo con los datos analizados, después de la firma del Trado de Libre Comercio de América del Norte (TLCAN), han mejorado significativamente las exportaciones de México, pero la balanza comercial del país, y por consecuencia el PIB, no se han visto favorecidos de acuerdo con las expectativas.

Para hacer las debidas comparaciones, haremos uso primero de la siguiente ecuación para obtener la elasticidad ingreso de las importaciones:

$$
\operatorname{LIMP}=\alpha \operatorname{LPIB} \pi \mathrm{e} €
$$

Los parámetros obtenidos de la ecuación con datos de la tabla 3, utilizando el programa Eviews, nos dan el siguiente resultado:

$$
\text { LIMP }=-25.11+3.38 \text { LPIB }
$$

La elasticidad ingreso de las importaciones es de 3.38 .

Tabla 3.

\begin{tabular}{|l|r|r|r|r|}
\hline \multicolumn{1}{|c|}{ Observaciones } & $\mathbf{1 5}$ & $\begin{array}{c}\text { Método: } \\
\text { mínimos } \\
\text { cuadrados }\end{array}$ & $\begin{array}{c}\text { Variable } \\
\text { dependiente } \\
\text { IMP }\end{array}$ & $\begin{array}{c}\text { Variables } \\
\text { independientes } \\
\text { PIB }\end{array}$ \\
\hline Variable & Coeficiente & Error estándar & Estadísticot & Probabilidad \\
\hline Origen & -24.57997 & 0.114084 & 29.07959 & .0000 \\
\hline PIB & 3.317527 & 1.024675 & -23.98806 & .0000 \\
\hline Media PIB & 5.21512 & - & - & - \\
\hline
\end{tabular}

\begin{tabular}{|l|l|l|l|}
\hline$R^{2}$ & 0.674471 & Media var depend & 7995.22 \\
\hline$R^{2}$ ajustada & 0.620216 & Estadístico F & 12.43153 \\
\hline Durbin Watson & 1.459315 & Probabilidad F & 0.001190 \\
\hline
\end{tabular}

Nota: Resultado obtenido con el programa Eviews.

Fuente: Elaboración propia.

Tabla 4.

\begin{tabular}{|l|c|c|}
\hline Año & $\begin{array}{c}\text { IMP } \\
\text { (miles de millones de } \\
\text { dólares) }\end{array}$ & $\begin{array}{c}\text { PIB } \\
\text { (miles de millones } \\
\text { de pesos) }\end{array}$ \\
\hline 1996 & 89.469 & 6427.559 \\
\hline 1997 & 109.802 & 6898.433 \\
\hline 1998 & 125.65 & 7072.2 \\
\hline 1999 & 142.289 & 7410.151 \\
\hline 2000 & 174.934 & 7711.444 \\
\hline 2001 & 168.662 & 7576.348 \\
\hline 2002 & 168.912 & 7670.056 \\
\hline
\end{tabular}




\begin{tabular}{|l|c|c|}
\hline Año & $\begin{array}{c}\text { IMP } \\
\text { (miles de millones de } \\
\text { dólares) }\end{array}$ & $\begin{array}{c}\text { PIB } \\
\text { (miles de millones } \\
\text { de pesos) }\end{array}$ \\
\hline 2003 & 170.777 & 7781.183 \\
\hline 2004 & 197.137 & 8133.313 \\
\hline 2005 & 222.343 & 8423.951 \\
\hline 2006 & 256.631 & 8765.547 \\
\hline 2007 & 282.604 & 9078.916 \\
\hline 2008 & 309.501 & 8985.252 \\
\hline 2009 & 234.901 & 8802.47 \\
\hline 2010 & 301.819 & 9191.549 \\
\hline
\end{tabular}

Fuente: Modificado de Inegi y Banxico (2012).

\section{Conclusiones}

Analizando los datos de la tabla 5, en la segunda columna tenemos el cociente de la tasa de exportaciones y la elasticidad ingreso de las importaciones; en la tercera columna, la tasa de cambio del PIB; y en la tercera, la balanza comercial de México. La ley de Thirlwall se da en 1999, 2000, 2004, 2005, 2006, 2007, 2008 y 2010, es decir, en $50 \%$ de estos años.
Se observa que en los años mencionados cada que excede el cociente tasa de exportaciones-elasticidad ingreso de las importaciones a la tasa de crecimiento del PIB, la balanza comercial del país es negativa. Por lo que podemos concluir, considerando el periodo de 1996-2010, que la ley de Thirlwall se da en México.

Tabla 5.

\begin{tabular}{|l|r|r|r|}
\hline \multicolumn{1}{|c|}{ Año } & \multicolumn{1}{c|}{$\mathbf{X} / \boldsymbol{\pi}$} & \multicolumn{1}{c|}{ TC PIB } & \multicolumn{1}{c|}{ BC } \\
\hline 1996 & 6.12 & 5.16 & 6531 \\
\hline 1997 & 4.45 & 2.52 & 624 \\
\hline 1998 & 1.89 & 4.78 & -7742 \\
\hline 1999 & 4.75 & 4.06 & -5583 \\
\hline 2000 & 6.52 & -1.75 & -8003 \\
\hline 2001 & -1.42 & 1.24 & -9954 \\
\hline 2002 & 0.43 & 1.45 & -7916 \\
\hline 2003 & 0.77 & 4.52 & -5623 \\
\hline 2004 & 4.14 & 3.68 & -8811 \\
\hline 2005 & 4.13 & 4.06 & -7586 \\
\hline
\end{tabular}




\begin{tabular}{|l|r|r|r|}
\hline \multicolumn{1}{|c|}{ Año } & \multicolumn{1}{c|}{$\mathbf{X} / \boldsymbol{\pi}$} & \multicolumn{1}{c|}{ TC PIB } & \multicolumn{1}{c|}{ BC } \\
\hline 2006 & 4.93 & 3.57 & -6133 \\
\hline 2007 & 2.59 & -1.02 & -10074 \\
\hline 2008 & 2.83 & -2.03 & -16700 \\
\hline 2009 & -7.18 & 4.42 & -4.926 \\
\hline 2010 & 8.47 & 3.6 & -2.96 \\
\hline
\end{tabular}

Fuente: Elaboración propia.

Tabla 6. Base de datos general

\begin{tabular}{|l|r|r|r|r|r|r|r|}
\hline \multicolumn{1}{|c|}{ Año } & \multicolumn{1}{c|}{ PIB } & \multicolumn{1}{c|}{ EXP X } & \multicolumn{1}{c|}{ IMP } & \multicolumn{1}{c|}{ T C de X } & \multicolumn{1}{c|}{$\boldsymbol{\pi}$ X $\mathbf{\pi}$} & T C de PIB \\
\hline 1996 & 6427.559 & 95.999 & 89.469 & 20.69 & 3.38 & 6.12 & 5.16 \\
\hline 1997 & 6898.433 & 110.431 & 109.802 & 15.03 & 3.38 & 4.45 & 2.52 \\
\hline 1998 & 7072.2 & 117.701 & 125.65 & 6.4 & 3.38 & 1.89 & 4.78 \\
\hline 1999 & 7410.151 & 136.391 & 142.289 & 16.07 & 3.38 & 4.75 & 4.06 \\
\hline 2000 & 7711.444 & 166.454 & 174.934 & 22.04 & 3.38 & 6.52 & -1.75 \\
\hline 2001 & 7576.348 & 158.442 & 168.662 & -4.81 & 3.38 & -1.42 & 1.24 \\
\hline 2002 & 7670.056 & 160.762 & 168.912 & 1.46 & 3.38 & 0.43 & 1.45 \\
\hline 2003 & 7781.183 & 164.922 & 170.777 & 2.59 & 3.38 & 0.77 & 4.52 \\
\hline 2004 & 8133.313 & 187.998 & 197.137 & 13.99 & 3.38 & 4.14 & 3.68 \\
\hline 2005 & 8423.951 & 214.233 & 222.343 & 13.95 & 3.38 & 4.13 & 4.06 \\
\hline 2006 & 8765.547 & 249.925 & 256.631 & 16.67 & 3.38 & 4.93 & 3.57 \\
\hline 2007 & 9078.916 & 271.875 & 282.604 & 8.78 & 3.38 & 2.59 & -1.02 \\
\hline 2008 & 8985.252 & 291.886 & 309.501 & 9.57 & 3.38 & 2.83 & -2.03 \\
\hline 2009 & 8802.47 & 229.975 & 234.901 & -24.27 & 3.38 & -7.18 & 4.42 \\
\hline 2010 & 9191.549 & 298.859 & 301.819 & 28.63 & 3.38 & 8.47 & 3.6 \\
\hline
\end{tabular}

Fuente: Modificado de Inegi, Banxico y Cepal (2010).

\section{Recomendaciones}

La política fiscal de México debe ser encauzada al incremento del PIB mediante la disminución de los impuestos, buscando principalmente el fomento de la exportación de bienes y servicios.

Una reforma fiscal y crediticia apoyará el crecimiento de las exportaciones mexicanas de bienes intermedios, así como de bienes de capital, ayudará a que disminuya la elasticidad ingreso de las exportaciones y se incremente la elasticidad ingreso de las exportaciones haciendo que mejore la balanza comercial del país y mediante el multiplicador externo se incremente el PIB.

La política mexicana en el tipo de cambio debe de estar encaminada a buscar una menor volatilidad en la moneda de México 
con relación a la de Estados Unidos, para evitar que ello repercuta en cambios de la balanza comercial con efectos a corto plazo.

Es necesario aprovechar la disminución del desempleo y la inflación en México para encauzar la producción de bienes y servicios de exportación y mejorar el resultado de la balanza comercial del país.

\section{Referencias bibliográficas}

Banco de México (2012).

Calderón Villarreal, C. y Hernández Bielma, L. (2011, enero-junio). El TLCAN, una forma de integración económica dualista: comercio externo e inversión extranjera directa. Revista Estudios Sociales, 19(37), 91-118. Recuperado de http:// ehis.ebscohost.com/ehost/pdfviewer/ pdfviewer?vid $=4 \&$ sid $=\mathrm{d} 7 \mathrm{a} 86152-\mathrm{e} 638$ 41c6-9a9f-88423d964009\%40sessionm gr112\&hid $=106$

Comisión Económica para América Latina y el Caribe (Cepal) (2012).

Cortés Fregoso, H. y Domínguez González, J. A. (2009, enero-junio). El PIB y la balanza comercial a 15 años del TLCAN. Revista Expresión Económica, XII (8), 112-122.
Flores Salgado, J. y Novelo Urdanivia, F. (2010). Hacia el mercado común norteamericano. Análisis Económico, XXV(58), 185-207. Recuperado de http:// ehis.ebscohost.com/ehost/pdfviewer/ pdfviewer?vid $=4 \& s i d=8 c 5 b 4405-720 c-$ 406b-8411-4433fabld6ac\%40sessionm gr110\&hid $=106$

García Molina, M. y Ruiz Tavera, J. K. (2009). Ley de Thirlwall y modelo de brechas: un modelo unificado. Documentos FCE, 5, 5 .

Guerrero de Lizardi, C. (2003). Modelo de crecimiento económico restringido por la balanza de pagos: evidencia para México, 1940-2000. El Trimestre económico, 70(278), 253-273.

Gujarati, D. N. (2004). Econometría (4. ${ }^{\mathrm{a}}$ ed.). México. McGraw-Hill.

Instituto Nacional de Estadística y Geografía (Inegi) (2012). México.

Loria, E.; Jesús, L. de y Brito, L. (2001). El desequilibrio comercial en México, ¿o porque ahora no podemos crecer al $7 \%$ ? Algunos elementos conceptuales. Momento económico, 16-21.

Pugel, T. A. (2004). Economía internacional. México. McGraw-Hill.

Thirlwall, A. (2003). La naturaleza del crecimiento económico. México: Fondo de Cultura Económica. 